\title{
El estudio de materiales de autoinstrucción como estrategia para incrementar el cumplimiento en la integración del expediente clínico.
}

\section{Artículo Original}

Saúl De los Santos-Briones ${ }^{1}$, Dulce Ma. Cruz-Lavadores ${ }^{1}$ Manuel de J. Chávez-Chan².

${ }^{1}$ Servicios de Salud de Yucatán, ${ }^{2}$ Programa de Desarrollo Humano Oportunidades, Instituto Mexicano del Seguro Social, Delegación Yucatán, Mérida, Yucatán, México.

\section{RESUMEN.}

Objetivo. Probar una estrategia de intervención para incrementar el nivel de cumplimiento de los criterios normativos establecidos para el llenado del expediente clínico en un centro de salud.

Material y Métodos. Se empleó un diseño cuasiexperimental. Se evaluaron 314 expedientes en la primera medición y 374 expedientes en la segunda, mediante una cédula de evaluación adaptada del Programa de Mejoría Continua de la Calidad de la Atención Médica de la Secretaría de Salud. La estrategia de intervención consistió en la autocapacitación del personal de salud mediante materiales de autoinstrucción. Se compararon el grado de conocimientos y el nivel de cumplimiento de la normatividad antes y después de la estrategia. Se empleó la prueba t de student para datos pareados y para datos no pareados como prueba estadística. Resultados. De acuerdo a la primera medición solamente en el $14.58 \%$ de las consultas que se cotejaron se llevó un registro de la atención médica en el expediente clínico; luego de la intervención el uso del expediente clínico se incrementó hasta un $63.90 \%$ de las consultas otorgadas. En todos los rubros evaluados sobre el llenado del expediente clínico se documentó un incremento en el cumplimiento de la normatividad correspondiente. El mayor cambio observado correspondió al rubro atención del adulto mayor (de 2.4 puntos de calificación obtenidos en promedio antes de la intervención a 6.29 puntos después, con una diferencia de 3.89 puntos; $\mathrm{p}=$ 0.0001). El menor cambio observado correspondió a notas médicas (de 7.72 puntos en promedio antes de la intervención a 8.61 después, con una diferencia de 0.97 puntos, $\mathrm{p}<0.0001$ ).

Conclusiones. El estudio de materiales de autoinstrucción demostró ser una estrategia efectiva para incrementar el nivel de cumplimiento de los criterios técnicos y normativos establecidos para el llenado del expediente clínico en la unidad sede de la

Solicitud de sobretiros: Dr. Saúl De los Santos Briones. Calle 67 No. 240 entre Av. 122 y Av. 128, Fraccionamiento Yucalpetén, C.P. 97248. Mérida, Yucatán, México. Correo electrónico: dlsbriones@yahoo.com Recibido el 19/Mayo/2003. Aceptado para publicación el 30/Octubre/2003. 


\section{S De los Santos-Briones, DM Cruz-Lavadores, M de J Chávez-Chan.}

investigación. (Rev Biomed 2003; 14:227-237)

Palabras clave: expediente clínico, estudios de intervención, calidad de atención médica.

\section{SUMMARY.}

The study of self-explanatory materials like strategy to increase the execution in the integration of the clinical file.

Objective. To test an intervention strategy in order to increase the level of execution of the established normative approaches for the handling of the clinical file in a health center.

Material and Methods. A quasi-experimental design was used. 314 files were evaluated in the first measurement and 374 files in the second, by means of a questionnaire adapted from the Program of Continuous Improvement of the Quality of Medical Attention of the Secretary of Health. The intervention strategy consisted in training health personnel by means of self-explanatory materials. The degree of knowledge and the level of execution of the normative approaches were compared before and after the strategy. The "t student" test for paired data and for unpaired data was used as a statistic test.

Results. According to the first measurement, only in $14.58 \%$ of the consultations was medical attention registered in the clinical file; after the intervention the use of the clinical file was increased until $63.90 \%$ of the granted consultations. An increment was documented in the execution of the established normative approaches in all the items evaluated. The biggest observed change corresponded to the elder attention item (from 2.4 points on the average before the intervention to 6.29 points later, with a difference of 3.89 points; $\mathrm{p}=0.0001$ ). The smallest observed change corresponded to medical notes (from 7.72 points before the intervention, to 8.61 later, with a difference of 0.97 points, $\mathrm{p}<0.0001$ ).

Conclusions. The study of self-explanatory materials demonstrated to be an effective strategy to increase the level of execution of the established technical and normative approaches for the registration of the clinical file in the unit headquarters of the investigation.

(Rev Biomed 2003; 14:227-237)

Key words: clinical file, intervention studies, quality in medical assistance.

\section{INTRODUCCIÓN.}

El expediente clínico se define como "el conjunto de documentos escritos, gráficos e imagenológicos, en los cuales el personal de salud hace los registros, anotaciones y certificaciones correspondientes a su intervención"(1). Los criterios para la integración y uso del expediente clínico se encuentran plasmados en la Norma Oficial Mexicana NOM-168-SSA11998, la cual constituye una herramienta de obligatoriedad para los sectores público, social y privado del Sistema Nacional de Salud (1).

Los primeros antecedentes relacionados con la evaluación de expedientes clínicos en nuestro país se encuentran en la década de los 50, cuando el Instituto Mexicano del Seguro Social (IMSS) creó la Comisión de Supervisión Médica e integró la auditoría médica, con lo que sentó las bases para la evaluación de la calidad de la atención médica y desarrolló modelos sistemáticos de evaluación de la misma. En la década de los 70, a partir de la experiencia del IMSS, adquirió mayor importancia la Auditoría Médica y se iniciaron acciones similares en el Instituto de Seguridad y Servicios Sociales para los Trabajadores del Estado (ISSSTE) y en el Sistema Nacional para el Desarrollo Integral de la Familia (DIF) (2).

En la Secretaría de Salud (SSA), no fue hasta 1997 cuando la Dirección General de Regulación de los Servicios de Salud creó en su estructura a la Dirección de Calidad de la Atención Médica, entre cuyas funciones se encontraron la coordinación y vigilancia sistemática del desarrollo de procedimientos tendientes a mejorar la calidad de la atención en el otorgamiento de los servicios, así como a elevar la satisfacción de los usuarios y de los prestadores mediante la ejecución de un programa diseñado ex profeso. Uno de los componentes del programa fue la evaluación de la elaboración del expediente clínico (2).

\section{Revista Biomédica}




\section{Cumplimiento en la integración del expediente clínico.}

La regulación del manejo del expediente clínico se orienta a permitir los usos médico, jurídico, de enseñanza e investigación, así como para fines de evaluación y control administrativo (1). Las acciones destinadas a sistematizar, homogeneizar y actualizar el manejo del expediente clínico han sido identificadas como promotoras del establecimiento de una cultura de la calidad en el proceso de atención médica ya que permite su empleo como instrumento para el estudio racional y la solución de los problemas de salud del usuario. Para el personal de salud se constituye en un instrumento de orientación, capacitación y apoyo técnico (3).

La evaluación del llenado del expediente clínico puede hacerse desde el enfoque de la estructura, el proceso y los resultados (4). El término estructura menciona todos los atributos -materiales y organizacionales-relativamente estables en los sitios en donde se proporciona la atención. El proceso incluye todo lo que médicos y otros proveedores de atención hacen por los pacientes, así como la habilidad con la cual lo llevan a cabo. Finalmente, el término resultado comprende lo que se ha obtenido para el paciente; específicamente supone un cambio en el estado de salud, para bien o para mal, e incluye otras consecuencias de la atención, por ejemplo el conocimiento adquirido sobre la enfermedad, y cambios en la conducta que conducen a la salud y la satisfacción del paciente $(4,5)$.

En este trabajo se presentan los resultados cuantitativos de una evaluación del proceso en el manejo del expediente clínico en un centro de salud urbano, realizada en el marco del Sistema de Apoyos a la Investigación Operativa (6). Observaciones preliminares efectuadas en la unidad sede de la investigación, refirieron un bajo cumplimiento de la normatividad referente a la integración, uso y manejo del expediente clínico, dificultando al personal de salud el seguimiento de los esquemas terapéuticos instaurados, así como también el seguimiento de las acciones preventivas y de rehabilitación, implicando para el personal directivo dificultades para su uso como instrumento de supervisión y evaluación.

Nuestro estudio se orientó a determinar en qué medida se llevaba a cabo el registro de la atención médica otorgada en lo expedientes clínicos de la unidad sede conforme a los criterios técnicos y normativos establecidos, así como probar una estrategia de intervención para incrementar el nivel de cumplimiento de dichos criterios, como medio para contribuir al desarrollo de una cultura de la calidad en la atención médica.

\section{MATERIAL Y MÉTODOS.}

El diseño correspondió a un estudio cuasiexperimental que incluyó tres etapas: una primera evaluación que proporcionó elementos diagnósticos sobre el estado inicial de la problemática en estudio, la introducción de la estrategia y, finalmente, una segunda medición para documentar el nivel de cambio observado.

La unidad médica sede de la investigación fue el centro de salud urbano de los Servicios de Salud de Yucatán en la Ciudad de Valladolid, al oriente del Estado de Yucatán, México. Esta unidad atiende a una población de influencia de 19,784 personas; está conformado por 6 núcleos básicos, brinda servicios de consulta externa, medicina preventiva y odontología. Su plantilla de personal está conformada por 36 personas entre las áreas administrativa, médica y paramédica.

Para la evaluación inicial se contó con un tamaño de muestra de 314 expedientes y para la evaluación final de 374 , con una potencia de $90 \%$, precisión \pm 0.05 , y a $=0.05$. El universo estuvo conformado por todos los expedientes clínicos en los que se llevó el registro de las consultas médicas que demandó la población del área de influencia. La selección de expedientes se llevó a cabo a partir de las consultas otorgadas en los meses de septiembre y diciembre de 2001 mediante un muestreo aleatorio simple. Se tuvo cuidado de que en cada muestra se incluyera una proporción de expedientes de cada consultorio acorde con su aporte a la productividad promedio mensual del centro de salud. Para tal efecto se fijó previamente la cuota de expedientes a evaluar por cada consultorio. En el cuadro 1 se presenta el número y el porcentaje correspondiente de expedientes evaluados de cada 
S De los Santos-Briones, DM Cruz-Lavadores, M de J Chávez-Chan.

Cuadro 1

Número de expedientes en los cuales se evaluaron los criterios técnicos y normativos establecidos para su llenado según consultorio y turno de labores. Centro de Salud Urbano de Valladolid, Yucatán. 2001-2002.

\begin{tabular}{|c|c|c|c|c|c|c|}
\hline \multirow[t]{2}{*}{ Consultorio } & \multicolumn{2}{|c|}{ Primera medición } & \multicolumn{2}{|c|}{ Segunda medición } & \multicolumn{2}{|c|}{ Total } \\
\hline & Número & $\%$ & Número & $\%$ & Número & $\%$ \\
\hline 1 & 58 & $18.47 \%$ & 60 & $16.04 \%$ & 118 & $17.15 \%$ \\
\hline 2 & 42 & $13.38 \%$ & 55 & $14.71 \%$ & 97 & $14.10 \%$ \\
\hline 3 & 56 & $17.83 \%$ & 62 & $16.58 \%$ & 118 & $17.15 \%$ \\
\hline 4 & 80 & $25.48 \%$ & 89 & $23.80 \%$ & 169 & $24.56 \%$ \\
\hline 5 & 64 & $20.38 \%$ & 71 & $18.98 \%$ & 135 & $19.62 \%$ \\
\hline 6 & 14 & $04.46 \%$ & 37 & $09.89 \%$ & 51 & $7.41 \%$ \\
\hline Total & 314 & $100 \%$ & 374 & $100 \%$ & 688 & $100 \%$ \\
\hline \multicolumn{7}{|l|}{ Turno de labores } \\
\hline Matutino & 236 & $75.15 \%$ & 266 & $71.12 \%$ & 502 & $72.96 \%$ \\
\hline Vespertino & 78 & $24.84 \%$ & 108 & $28.87 \%$ & 186 & $27.03 \%$ \\
\hline Total & 314 & $100 \%$ & 374 & $100 \%$ & 688 & $100 \%$ \\
\hline
\end{tabular}

consultorio en la primera y segunda medición. Para poder seleccionar las muestras en la primera medición se cotejaron en total 3009 consultas, en tanto que en la segunda medición se cotejaron 2842 consultas.

La estrategia de intervención estuvo dirigida a incrementar y uniformar el conocimiento de los integrantes de los equipos de salud de cada consultorio en lo que se refiere a los criterios técnicos que norman la integración y el llenado del expediente clínico. La estrategia de intervención probó adicionalmente una modalidad para la capacitación del personal en sus propios centros de labores: la introducción de materiales educativos para la autoinstrucción del personal de salud. Para tal efecto se elaboró el cuaderno "Integración, Uso y Manejo del Expediente Clínico. Material para la Autoinstrucción del personal de salud". Los cuadernos fueron distribuidos entre el personal asignado a los consultorios del centro de salud para su estudio. Antes y después del estudio del material de autoinstrucción se llevaron a cabo evaluaciones del grado de conocimientos sobre el tema. La estrategia de intervención se aplicó en octubre de 2001.

De los 20 trabajadores que conformaban los 6 núcleos básicos del centro de salud urbano al momento de la intervención, 18 recibieron el material de autocapacitación y fueron evaluados (90\%). Los 2 restantes se encontraban disfrutando de su período vacacional en el momento de distribuir el material y aplicar la evaluación inicial. No obstante que no se consideró su participación en la evaluación inicial y final, a su reincorporación se les proporcionó el material de autoinstrucción. No hubo pérdidas en el seguimiento, por lo que los 18 sujetos que ingresaron al estudio continuaron y concluyeron la fase de intervención. De los sujetos que intervinieron, 13 de ellos (72.22\%) laboraban en el turno matutino y los 5 restantes $(27.78 \%)$ en el vespertino. De las personas que fueron evaluadas, 5 correspondieron a médicos, 7 a enfermeras de campo o promotores y 6 a enfermeras de área clínica.

Los instrumentos para la recolección de información fueron: un cuestionario para la evaluación del grado de conocimientos sobre la Norma Oficial Mexicana para la integración y uso del expediente clínico y la cédula de evaluación del cumplimiento de los criterios técnico-normativos para la integración y uso del expediente clínico.

La evaluación del grado de cumplimiento de los criterios normativos para el llenado del expediente

\section{Revista Biomédica}




\section{Cumplimiento en la integración del expediente clínico.}

Cuadro 2

Aspectos evaluados sobre el manejo del expediente clínico.

\begin{tabular}{|c|c|c|}
\hline \multirow{11}{*}{$\begin{array}{l}\text { Aspectos } \\
\text { generales } \\
\text { del } \\
\text { expediente } \\
\text { clínico }\end{array}$} & Carpeta & $\begin{array}{l}\text { ¿Cuenta con la carpeta familiar? } \\
\text { ¿Se encuentra consignada la información requerida en la carpeta familiar? }\end{array}$ \\
\hline & \multirow[t]{2}{*}{ Historia Clínica Individual } & ¿Cuenta con historias clínicas individuales, para cada miembro de la familia? \\
\hline & & ¿Se encuentra consignada la información requerida en las historias clínica individuales? \\
\hline & \multirow[t]{2}{*}{ Evaluación de Riesgos } & ¿Cuenta con la hoja de evaluación de riesgos? \\
\hline & & ¿Se encuentra consignada la información requerida en la hoja de evaluación de riesgos? \\
\hline & \multirow[t]{2}{*}{ Microdiagnóstico } & ¿Cuenta con la cédula de microdiagnóstico familiar? \\
\hline & & ¿Se encuentra consignada la información requerida en la cédula de microdiagnóstico familiar? \\
\hline & \multirow[t]{4}{*}{ Apgar } & ¿Cuenta con la hoja de Apgar Individual? \\
\hline & & ¿Se encuentra consignada la información requerida en la hoja de Apgar Individual? \\
\hline & & ¿Cuenta con la hoja de Apgar Familiar Anual? \\
\hline & & ¿Se encuentra consignada la información requerida en la hoja de Apgar Familiar Anual? \\
\hline \multirow[t]{14}{*}{ Notas médicas } & \multirow[t]{14}{*}{ Notas médicas } & ¿Cuenta con la nota médica de consulta? \\
\hline & & ¿La nota médicas se encuentran dispuestas siguiendo un orden cronológico? \\
\hline & & ¿Se encuentra consignada la fecha de la consulta? \\
\hline & & ¿Se encuentra consignada la hora de la consulta? \\
\hline & & ¿Se encuentra consignado el peso del paciente? \\
\hline & & ¿Se encuentra consignada la estatura del paciente? \\
\hline & & ¿Se encuentra consignado el motivo de la consulta? \\
\hline & & ¿Se encuentran consignados los hallazgos de la exploración física? \\
\hline & & ¿Se encuentra consignada la impresión diagnóstica? \\
\hline & & ¿Se encuentra consignado el tratamiento establecido? \\
\hline & & ¿Está consignado el nombre del médico, al final de la nota médica? \\
\hline & & ¿Está consignada la firma del médico, al final de la nota médica? \\
\hline & & ¿Se observa limpieza y pulcritud en la nota médica? \\
\hline & & ¿Se observa legibilidad en la nota médica? \\
\hline \multirow{25}{*}{$\begin{array}{l}\text { Aspectos } \\
\text { específicos } \\
\text { del } \\
\text { expediente } \\
\text { clínico }\end{array}$} & \multirow[t]{5}{*}{ Menor de 5 años } & ¿La consulta corresponde a un menor de 5 años en la familia?* \\
\hline & & ¿Cuenta con la hoja de atención integral del menor? \\
\hline & & ¿Se encuentra consignada la información requerida en la hoja de atención integral del menor? \\
\hline & & ¿Cuenta con la hoja de vigilancia del estado nutricional? \\
\hline & & ¿Se encuentra consignada la información requerida en la hoja de vigilancia nutricional? \\
\hline & \multirow[t]{3}{*}{ Escolares } & ¿La consulta corresponde a un escolar de entre 5 y 14 años de edad?* \\
\hline & & ¿Cuenta con hoja de atención del escolar? \\
\hline & & ¿Se encuentra consignada la información requerida en la hoja de atención del escolar? \\
\hline & \multirow[t]{3}{*}{ Mujeres en edad fértil } & ¿La consulta corresponde a una mujer en edad fértil?** \\
\hline & & ¿Cuenta con hoja de atención integral a la mujer en edad fértil? \\
\hline & & $\begin{array}{l}\text { ¿Se encuentra consignada la información requerida en la hoja de atención integral a la } \\
\text { mujer en edad fértil? }\end{array}$ \\
\hline & \multirow[t]{3}{*}{ Planificación Familiar } & ¿La consulta corresponde a una mujer en edad fértil con vida sexual activa?* \\
\hline & & ¿Cuenta con historia clínica de PF? \\
\hline & & ¿Se encuentra consignada la información requerida en la historia clínica de PF? \\
\hline & \multirow[t]{5}{*}{ Control Prenatal } & ¿La consulta corresponde a una mujer embarazada?* \\
\hline & & ¿Cuenta con historia clínica perinatal? \\
\hline & & ¿Se encuentra consignada la información requerida en la historia clínica perinatal? \\
\hline & & ¿Cuenta con curva de ganancia de altura uterina? \\
\hline & & ¿Se encuentra consignada la información requerida en la curva de ganancia de altura uterina? \\
\hline & \multirow[t]{3}{*}{ Adulto } & ¿La consulta corresponde a un adulto?* \\
\hline & & ¿Cuenta con hoja de atención integral al adulto? \\
\hline & & ¿Se encuentra consignada la información requerida en la hoja de atención integral al adulto? \\
\hline & \multirow[t]{3}{*}{ Adulto Mayor } & ¿La consulta corresponde a un adulto mayor de 65 años?* \\
\hline & & ¿Cuenta con hoja de atención integral al adulto mayor? \\
\hline & & ¿Se encuentra consignada la información requerida en la hoja de atención integral al adulto mayor? \\
\hline
\end{tabular}

* Corresponden a preguntas filtro que no recibieron puntuación. 


\section{S De los Santos-Briones, DM Cruz-Lavadores, M de J Chávez-Chan.}

clínico, se llevó a cabo mediante una cédula adaptada, que combinó los lineamientos establecidos en la Norma Oficial Mexicana (NOM) 168-SSA1-1998 (1) así como en la Guía Metodológica del Modelo de Atención a la Salud para Población Abierta (7); así mismo, su diseño tomó elementos de un formato de evaluación del expediente clínico establecido en el Programa de Mejoría Continua de la Calidad de la Atención Médica de la SSA de 1998 (2). La cédula estuvo conformada por 9 rubros agrupados en tres apartados: aspectos generales del expediente clínico, notas médicas y aspectos específicos del expediente clínico.

El apartado de aspectos generales del expediente clínico se evaluó mediante 12 reactivos si se contaba con la carpeta familiar, las historias clínicas individuales, las hojas de evaluación de riesgos, la hoja de microdiagnóstico, la hoja de apgar individual, la hoja de apgar familiar anual, así como el grado en que se encontraba consignada la información solicitada en cada una de ellas. Dependiendo de la pregunta planteada cada reactivo se pudo calificar optando por una escala nominal en las que las posibles respuestas eran sí, parcialmente, o no o bien una escala ordinal en la cual las posibles respuestas eran de 0 a $33 \%$ de las veces, de 34 a $66 \%$ de la veces y de 67 a $100 \%$ de las veces, respectivamente. En este apartado a cada repuesta sí o de 67 a 100\% de la veces correspondió una calificación de 0.83 puntos; a la respuesta parcialmente o de 34 a $66 \%$ de la veces correspondió una calificación de 0.41 puntos y a cada respuesta no ó 0 a 33\% de las veces correspondió una calificación de 0 puntos. De esta forma, dependiendo de las respuestas obtenidas este apartado pudo ser evaluado en cada caso, con una calificación que podría ir desde 0 hasta 9.9 puntos.

El apartado de notas médicas se evaluó mediante 14 reactivos que cuestionaban si se contaba con ella, si estaban dispuestas en orden cronológico, si consignaba la fecha, la hora, la estatura, el peso, el motivo de consulta, los hallazgos a la exploración, la impresión diagnóstica, el tratamiento establecido, el nombre y la firma del médico, así como si se observaba limpieza, pulcritud y legibilidad en la nota.
Dependiendo de la pregunta planteada se pudo optar por las escalas señaladas en cuyo caso la puntuación se asignó de la siguiente manera: a cada repuesta sí o de 67 a 100\% de la veces correspondió una calificación de 0.71 puntos; a cada respuesta parcialmente o de 34 a $66 \%$ de la veces correspondió una calificación de 0.35 puntos; a cada respuesta no ó 0 a $33 \%$ de las veces correspondió una calificación de 0 puntos. Dependiendo de las respuestas obtenidas este apartado también pudo ser evaluado en cada caso con una calificación que podría ir desde 0 hasta 9.9 puntos.

Finalmente, en el apartado de aspectos específicos del expediente clínico se evaluaron por separado 7 rubros, cada uno de ellos correspondiente a un programa sanitario específico; estos programas fueron atención del menor de 5 años, atención del escolar, mujer en edad fértil, control prenatal, planificación familiar, atención del adulto y atención del adulto mayor. Las escalas de medición empleadas fueron las mimas. Para los programas de atención del menor de 5 años y control prenatal la puntuación asignada a cada repuesta sí o de 67 a 100\% de la veces correspondió una calificación de 2.5 puntos, a la respuesta parcialmente o de 34 a $66 \%$ de la veces correspondió una calificación de 1.25 puntos, y a cada respuesta no ó 0 a 33\% de las veces correspondió una calificación de 0 puntos. Dependiendo de las respuestas obtenidas, estos apartados pudieron recibir una calificación que podría ir desde 0 hasta 10.0 puntos. Para los programas de atención del escolar, mujer en edad fértil, control prenatal, planificación familiar, atención del adulto y atención del adulto mayor la puntuación asignada a cada repuesta sí o de 67 a $100 \%$ de la veces correspondió una calificación de 5.0 puntos, a la respuesta parcialmente o de 34 a $66 \%$ de la veces correspondió una calificación de 2.5 puntos y a cada respuesta no ó 0 a $33 \%$ de las veces correspondió una calificación de 0 puntos. En estos rubros las calificaciones otorgadas también pudieron ir de 0 hasta 10.0 puntos. En el cuadro 2 se presentan los aspectos evaluados sobre el manejo del expediente clínico.

Esta cédula fue sometida a una prueba piloto en

\section{Revista Biomédica}




\section{Cumplimiento en la integración del expediente clínico.}

la que se evaluó su confiabilidad y validez; también se probó la cédula para determinar el grado de conocimiento sobre los criterios normativos establecidos para el llenado del expediente clínico, en la cual se examinó la claridad de las preguntas formuladas en la cédula.

Conforme a las etapas del estudio, se tuvieron dos variables dependientes: a) grado de conocimientos sobre la NOM para la integración y uso del expediente clínico, y b) calificación obtenida en la evaluación sobre el nivel de cumplimiento de la norma oficial. Estas variables constituyen los indicadores a ser evaluados en la primera y segunda medición. La variable independiente fue haber participado en la estrategia de intervención.

La captura de datos se realizó en el programa EpiInfo 6.0. El análisis estadístico se realizó con el programa Stata 6.0. Se calcularon porcentajes y medias con intervalos de confianza del $95 \%$. Se empleó la t de student para datos no pareados y muestras independientes, para comparar el cumplimiento de los criterios técnico-normativos del expediente clínico, antes y después de la intervención. La comparación del grado de conocimientos sobre la normatividad en el manejo del expediente antes y después del estudio del material de autoinstrucción se evaluó mediante la prueba de $t$ de student para datos pareados.

\section{RESULTADOS.}

El 83\% del personal encuestado previamente a la instrumentación de la estrategia de intervención señaló que no había recibido capacitación sobre el uso y llenado del expediente clínico, mientras que el $16.67 \%$ declaró que sí la había recibido. Entre quienes refirieron que sí la recibieron señalaron que había ocurrido en promedio 1 año 6 meses antes, con un rango de 5 meses hasta 3 años.

El 66\% de los sujetos señaló que nunca había leído la Guía Metodológica sobre el Modelo de Atención a la Salud para Población Abierta (MASPA). Quienes sí lo habían hecho, lo hicieron en promedio 2 años 1 mes antes de la encuesta, con un rango de 6 meses hasta 5 años.

También, una proporción alta de los sujetos en estudio (83.33\%) declaró no haber leído nunca la Norma Oficial Mexicana NOM 168-SSA1-1998 sobre el manejo e integración del expediente clínico. Entre quienes lo hicieron, había ocurrido en promedio 9 meses antes, con un rango entre 5 meses y 1 año.

El 88.89\% de los sujetos que participaron en la intervención declararon haber estudiado el material de autoinstrucción conforme a las recomendaciones. Hubo 2 sujetos que reportaron no haberlo hecho; en ambos casos se adujo no haber tenido tiempo para hacerlo.

En la evaluación previa al estudio del material de autoinstrucción la puntuación promedio obtenida sobre el grado de conocimiento fue de 48.83 puntos (mínimo $=35$, máximo $=80$; desviación estándar $=10.62$; $\mathrm{IC}=43.54,54.11)$. En la evaluación posterior, la puntuación promedio obtenida fue de 72.55 puntos $($ mínimo $=43$, máximo $=93$; desviación estándar $=$ 15.57; $\mathrm{IC}=64.80,80.30$ ). Se documentó un incremento promedio de 23.72 puntos en el conocimiento de los criterios técnicos establecidos para el manejo del expediente clínico. Este incremento resultó estadísticamente significativo $(\mathrm{p}=0.0000)$. El mayor incremento registrado en un individuo fue de 47.0 puntos (de 43 puntos en el pretest a 90 puntos en el postest). Los menores incrementos observados fueron de sólo 2.0 puntos y ocurrieron en 2 sujetos que reportaron no haber estudiado el material de autoinstrucción.

De las 3009 consultas cotejadas en la primera medición se encontró que el registro de la atención médica en el expediente clínico ocurrió sólo en 439 consultas (el $14.58 \%$ de los casos). En la segunda medición, en la cual se cotejaron 2842 consultas, se documentó que en 1816 casos (63.9\%) se llevó un registro de la atención en el expediente clínico, con independencia de la calidad de la información consignada. El cambio por consultorio en el porcentaje de uso del expediente clínico como resultado de la intervención, sin considerar la calidad de la información consignada, se presenta en el cuadro 3.

En la medición inicial de los registros hechos en el expediente clínico la puntación más alta correspondió al rubro de registro de la nota médica, con 7.72 puntos

Vol.14/No.4/Octubre-Diciembre, 2003 
S De los Santos-Briones, DM Cruz-Lavadores, M de J Chávez-Chan.

Cuadro 3

Cambio observado en el porcentaje de uso del expediente clínico antes y después de la intervención*. Centro de Salud Urbano de Valladolid, Yucatán. 2001-2002.

\begin{tabular}{lcc}
\hline Consultorio & \multicolumn{2}{c}{$\begin{array}{c}\text { Porcentaje de uso del expediente clínico } \\
\text { Después de la intervención }\end{array}$} \\
\hline Consultorio 1 & $23.83 \%$ & $83.44 \%$ \\
Consultorio 2 & $12.00 \%$ & $65.07 \%$ \\
Consultorio 3 & $13.59 \%$ & $68.26 \%$ \\
Consultorio 4 & $14.14 \%$ & $49.33 \%$ \\
Consultorio 5 & $13.86 \%$ & $52.60 \%$ \\
Consultorio 6 & $10.12 \%$ & $64.70 \%$ \\
\hline Total & $14.58 \%$ & $63.90 \%$ \\
\hline
\end{tabular}

* Sin tomar en cuenta la calidad de la información consignada.

en promedio. La calificación más baja correspondió al rubro del registro de la atención otorgada al adulto mayor, con 2.4 puntos en promedio. En el registro de la atención otorgada en los rubros de atención prenatal y planificación familiar se obtuvo puntuaciones de 7.01 y 7.63 en promedio, respectivamente. El registro de la atención del menor de 5 años alcanzó una calificación de 6.04 puntos en promedio, en los expedientes evaluados.

En la segunda medición los promedios de puntuación más altos documentados fueron para los rubros de atención del menor de 5 años (8.94 puntos), notas médicas (8.70 puntos) y control prenatal (8.61 puntos). Los promedios de puntuación más bajos documentados ocurrieron en los rubros de atención del adulto (6.16 puntos) y atención del adulto mayor (6.29 puntos). En todos los rubros, el cambio de puntuación promedio documentado antes y después de la intervención obtuvo significancia estadística, exceptuando el registro de la atención de planificación familiar, en la cual la diferencia de puntuación promedio antes y después fue de sólo 0.24 puntos ( $\mathrm{p}=0.3925$ ). El cambio de mayor magnitud observado fue en el rubro atención del adulto mayor con una diferencia de 3.89 en la puntación promedio antes y después de la intervención ( $\mathrm{p}=0.0001$ ). En el cuadro 4 se resumen las puntuaciones logradas para cada uno de los aspectos evaluados antes y después de la intervención.

\section{DISCUSIÓN.}

El estudio exploratorio permitió documentar que el registro de la atención médica otorgada en los expedientes clínicos en la unidad sede, ocurría muy por debajo de nuestras estimaciones iniciales. No se generó un registro del padecimiento, hallazgos físicos y tratamiento instaurado en casi el $85 \%$ de las consultas otorgadas, con las correspondientes implicaciones que significan para la calidad de la atención médica que se brinda en la unidad. También se destaca el hecho de que una alta proporción de los sujetos en estudio no habían recibido capacitación formal sobre el manejo del expediente clínico: el 83\% no había recibido capacitación, el $66 \%$ no había leído la Guía Metodológica sobre el Modelo de Atención a la Salud para Población Abierta y el 83.33\% no había leído la Norma Oficial Mexicana correspondiente.

Las intervenciones para la resolución de problemas en las organizaciones de salud pueden abordar uno o varios de los factores contribuyentes $(8,9)$. En relación con las causas de las deficiencias en la calidad en el modelo de atención a la salud, se afirma categóricamente que los factores fundamentales de la calidad son inherentes al diseño de los sistemas y procesos. Los factores identificados pueden también ser clasificados en términos de estructura, proceso y resultado, siguiendo a Donabedian (10). En función del tiempo y recursos disponibles, nuestro proyecto

\section{Revista Biomédica}


Cumplimiento en la integración del expediente clínico.

\begin{tabular}{|c|c|c|c|c|c|c|c|c|}
\hline \multirow[t]{2}{*}{ Aspecto evaluado } & \multirow[b]{2}{*}{$\mathbf{n}$} & \multicolumn{2}{|c|}{$\begin{array}{l}\text { Antes de la } \\
\text { Intervención }\end{array}$} & \multicolumn{3}{|c|}{$\begin{array}{l}\text { Después de la } \\
\text { intervención }\end{array}$} & \multirow[t]{2}{*}{ Diferencia } & \multirow[t]{2}{*}{$\mathbf{p}$} \\
\hline & & $\overline{\mathbf{X}}$ & IC $(95 \%)$ & $\mathbf{n}$ & $\overline{\mathbf{X}}$ & IC $(95 \%)$ & & \\
\hline $\begin{array}{l}\text { Aspectos generales } \\
\text { del expediente clínico }\end{array}$ & 314 & 5.09 & $4.90,5.29$ & 374 & 7.14 & $7.04,7.23$ & 2.04 & 0.0000 \\
\hline Notas médicas & 229 & 7.72 & $7.58,7.86$ & 374 & 8.70 & $8.61,8.78$ & 0.97 & 0.0000 \\
\hline $\begin{array}{l}\text { Atención del menor } \\
\text { de cinco años }\end{array}$ & 41 & 6.04 & $5.31,6.77$ & 78 & 8.94 & $8.63,9.25$ & 2.89 & 0.0000 \\
\hline Atención del escolar & 14 & 4.64 & $3.87,5.41$ & 36 & 7.22 & $6.36,8.07$ & 2.57 & 0.0004 \\
\hline $\begin{array}{l}\text { Atención de la mujer } \\
\text { en edad fértil }\end{array}$ & 122 & 3.35 & $2.85,3.85$ & 146 & 6.59 & $6.13,7.05$ & 3.23 & 0.0000 \\
\hline $\begin{array}{l}\text { Atención de planifi- } \\
\text { cación familiar }\end{array}$ & 38 & 7.63 & $6.35,8.91$ & 47 & 7.87 & $6.65,9.08$ & 0.24 & 0.3925 \\
\hline Control prenatal & 44 & 7.01 & $6.35,7.67$ & 44 & 8.61 & $8.05,9.16$ & 1.59 & 0.0002 \\
\hline Atención del adulto & 50 & 3.55 & $2.87,4.22$ & 56 & 6.16 & $5.59,6.73$ & 2.61 & 0.0000 \\
\hline $\begin{array}{l}\text { Atención del adulto } \\
\text { mayor }\end{array}$ & 18 & 2.4 & $1.15,3.64$ & 27 & 6.29 & $4.88,7.70$ & 3.89 & 0.0001 \\
\hline
\end{tabular}

$\mathrm{n}=$ número de expedientes, $\overline{\mathrm{X}}=$ media, $\mathrm{IC}=$ intervalo de confianza.

intentó intervenir en un factor de proceso que determina el incumplimiento del llenado del expediente clínico: la falta de capacitación del personal de salud.

En particular, nuestra propuesta consistió en la elaboración de materiales propios para la autoinstrucción del personal de salud. Los materiales de autoinstrucción son documentos escritos o gráficos que contienen la información relevante para normar una conducta, que pueden ser autoestudiados por el personal a quien va dirigido en los tiempos y espacios que mejor se adapten a sus actividades y cuyo aprendizaje puede ser objeto de evaluación.

Esta modalidad de capacitación requiere una definición precisa del contenido temático sobre el que se requiere capacitar, así como de una evaluación de la viabilidad de que los contenidos sean abordados mediante la autoinstrucción. Implica que el personal responsable del programa de capacitación defina con precisión el contenido, la extensión y la profundidad de los tópicos a tratar, así como la pertinencia de establecer una serie de indicadores y su estándar mediante los cuales serán evaluados y, desde luego, deberán incorporarse diversos materiales de apoyo como videos, trípticos, folletos, manuales, cintas de audio, etc. Se requiere, así mismo, emplear formatos de redacción y estilo que favorezcan la comprensión y estudio de los contenidos.

El proceso descrito fue seguido para el caso de la necesidad particular en nuestro proyecto de investigación: incrementar el cumplimiento de los criterios normativos para el uso y manejo del expediente clínico. Desde luego, se partió de la observación de que la falta de capacitación del personal operativo era un factor determinante del bajo nivel de cumplimiento en el llenado e integración del expediente clínico en el centro de salud urbano de Valladolid.

La proporción de expedientes evaluados correspondientes a cada tipo de atención fue muy similar en cada medición, con lo que la posibilidad de 


\section{S De los Santos-Briones, DM Cruz-Lavadores, M de J Chávez-Chan.}

un sesgo en la selección de los expedientes que ingresaron al estudio quedó bajo control. Logró documentarse un incremento de 49.32 puntos porcentuales en el porcentaje de expedientes usados entre la primera y segunda medición. Esto significó un incremento de 4.3 veces en el porcentaje de expedientes en los cuales se llevó un registro escrito de la atención médica otorgada como resultado de la intervención y sin tomar en cuenta la calidad del registro.

Ahora bien, al evaluar la calidad de las anotaciones las calificaciones más altas en la medición inicial correspondieron a las notas médicas, atención de planificación familiar y control prenatal. Esto podría reflejar el hecho de que los mecanismos de supervisión se encaminan principalmente a estos rubros, por considerarlos más relevantes. La atención del adulto, del adulto mayor y de la mujer en edad fértil por su parte reportaron las menores calificaciones iniciales. Fueron precisamente en estos rubros en los que el cambio observado en la puntuación fue mayor.

El diseño del estudio, en el que no se contó con grupo control, limita la extrapolación de los resultados obtenidos a otros contextos. No obstante, la naturaleza de la investigación operativa de servicios de salud (6), orientada a la resolución de problemas concretos en los procesos de atención médica, justificó el diseño y permitió documentar la resolución de la problemática que dio origen a la investigación. Es recomendable, no obstante, llevar a cabo una tercera medición a fin de evaluar la permanencia en el tiempo del cambio generado. Así, la característica estática tradicional de las formas de control se dinamiza a través de la monitoría, entendida ésta como el seguimiento y la utilización de mediciones repetidas que nos permitan conocer el comportamiento de la calidad, detectar oportunamente desviaciones y, de esta manera, realizar las modificaciones pertinentes $(11,12)$.

Finalmente, es importante tener en cuenta las posibles desventajas de los materiales de autoestudio, como son la elevada inversión de tiempo en su elaboración, la necesidad de adecuar el material al perfil profesional del destinatario (13) y que puede ocurrir que el personal de salud no muestre la

\section{Revista Biomédica}

disposición necesaria para el estudio del material (14). Sobre este último aspecto, en nuestro estudio 2 personas, que representaron el $11.1 \%$ de quienes participaron en la estrategia, declararon no haber estudiado el material. Se señaló falta de tiempo y baja disposición para su estudio.

La posibilidad de falta de estudio podría ser disminuida si la acreditación del autoestudio del material formara parte del sistema de créditos de capacitación de la institución, logrando así incrementar el grado de satisfacción en los prestadores de la atención médica.

La satisfacción de las necesidades de capacitación y formación es esencial para que los prestadores directos de la atención médica se encuentren en condiciones de dar cumplimiento a los ordenamientos legales. El estudio de materiales de autoinstrucción demostró ser una estrategia efectiva para incrementar el nivel de conocimientos y el nivel de cumplimiento de los criterios técnicos y normativos establecidos para el llenado del expediente clínico en la unidad sede del proyecto. Además de cubrir las necesidades de capacitación del personal, esta modalidad permite que el personal continúe cumpliendo sus funciones y demuestra ser efectiva, eficiente y de calidad. Efectiva porque documenta que logra su objetivo: incrementar los conocimientos y capacidades del personal y que se traduce en una modificación de la conducta. Eficiente porque cumple su objetivo a un menor costo. De calidad porque se adapta a las necesidades y expectativas del usuario. Una capacitación efectiva, eficiente y de calidad la podemos relacionar con satisfacción en el prestador, contribuyendo a fortalecer la cultura de calidad en la institución.

\section{AGRADECIMIIENTOS.}

Este trabajo de investigación se llevó a cabo con financiamiento del Sistema de Apoyos a la Investigación Operativa en Calidad, instrumentado por la Dirección General de Calidad y Educación en Salud de la Subsecretaría de Planeación e Innovación de la Secretaría de Salud Federal de México, instancias a las cuales hacemos expreso nuestro agradecimiento. 


\section{Cumplimiento en la integración del expediente clínico.}

\section{REFERENCIAS.}

1.- Secretaría de Salud. Compendio normativo para la prestación de servicios de salud. México: Secretaría de Salud; 2000.

2.- Poder Ejecutivo (México). Diario Oficial de la Federación. Norma Oficial Mexicana NOM-168 SSA1-1998 del expediente clínico. México: Secretaría de Salud; 1999.

3.- Aguirre-Gas H. Administración de la calidad de atención médica. Rev Med IMSS 1997; 35: 257-64

4.- Donabedian A. Garantía y monitoría de la calidad de la atención médica. Un texto introductorio. México: Instituto Nacional de Salud Pública. Perspectivas en Salud Pública; 1990.

5.- Donabedian A. La calidad de la atención médica. Definición y métodos de evaluación. México: Ediciones Científicas La Prensa Médica Mexicana; 1984.

6.- Secretaría de Salud. Modelo de investigación operativa de servicios de salud [material en CD ROM]. México, D.F.: Secretaría de Salud; 1998.

7.- Servicios Coordinados de Salud Pública en el Estado de Yucatán. Modelo de atención a la salud para población abierta. Guía metodológica. Mérida: Gobierno del Estado de Yucatán; 1996.

8.- Secretaría de Salud. Programa de mejoría continua de la calidad de la atención médica. Instrumentos de medición para primer nivel de atención [material en CD ROM]. México: Secretaría de Salud; 1998.

9.- Ruelas-Barájas E, Querol J. Calidad y eficiencia en las organizaciones de atención a la salud. México: FUNSALUD; 1994.

10.- Donabedian A. Continuidad y cambio en la búsqueda de la calidad. Salud Pública Mex 1993; 35: 238-47.

11.- Martínez-Ramírez A, Guzmán-Bihouet B, Ruiz-Orozco P, Van Dick-Puga M, León-Trujillo H, Martínez-Flores A. Evaluación de la calidad de la atención a la salud mediante indicadores y su estándar. Rev Med IMSS 1998; 36:487-96.

12.- Ramírez-Sánchez TJ, Nájera-Aguilar P, Nigenda-López G. Percepción de la calidad de la atención de los servicios de salud en México: perspectiva de los usuarios. Salud Pública Mex 1998; 40:3-12.
13.- Artaza O, Cabello X, Cortés V, Fuentes A, Guerrero JL, Mora ML, et al. Hospital Luis Calvo Mackenna: La calidad y la satisfacción usuaria, pilares estratégicos de cambio. Cuad Med Soc 1997; 38: 41-7.

14.- Aguirre-Gas H. Evaluación de la atención médica. Expectativas de pacientes y trabajadores. Salud Pública Mex 1990; 32: 170-80. 